\title{
One-Cycle Control Strategy for Dual-Converter Three-Phase PWM Rectifier under Unbalanced Grid Voltage Conditions
}

\author{
You Xu' ${ }^{\dagger}$, Qingjie Zhang*, and Kai Deng ${ }^{* *}$ \\ †School of Automation, Nanjing Institute of Technology, Nanjing, China \\ * College of Engineering, Nanjing Agriculture University, Nanjing, China \\ ${ }^{* *}$ School of Electrical Engineering, Southeast University, Nanjing, China
}

\begin{abstract}
In this paper, a dual-converter three-phase pulse width modulation (PWM) rectifier based on unbalanced one-cycle control (OCC) strategy is proposed. The proposed rectifier is used to eliminate the second harmonic waves of DC voltage and distortion of line currents under unbalanced input grid voltage conditions. The dual-converter PWM rectifier employs two converters, which are called positive-sequence converter and negative-sequence converter. The unbalanced OCC system compensates feedback currents of positive-sequence converter via grid negative-sequence voltages, as well as compensates feedback currents of negative-sequence converter via grid positive-sequence voltages. The AC currents of positive- and negative-sequence converter are controlled to be symmetrical. Thus, the workload of every switching device of converter is balanced. Only one conventional PI controller is adopted to achieve invariant power control. Then, the parameter tuning is simplified, and the extraction for positive- and negative-sequence currents is not needed anymore. The effectiveness and the viability of the control strategy are demonstrated through detailed experimental verification.
\end{abstract}

Key words: Invariant power control, Negative-sequence converter, One-cycle control, Positive-sequence converter, Voltage sag

\section{INTRODUCTION}

Public power systems are frequently under unbalanced voltage conditions, and single-phase grid voltage sag happens occasionally. Moreover, even harmonic waves appear on DC voltage, as well as distortion on line currents of traditional three-phase PWM rectifiers. Therefore, public power systems could be polluted by harmonics, and power loss of PWM rectifier could be increased dramatically. New control strategy and topology for three-phase PWM rectifier under unbalanced input voltage conditions must be developed. The control goal is to keep DC voltage constant and sinusoidal currents symmetrical together with unity-power factor. Several control methods have been reported for three-phase

Manuscript received Apr. 27, 2014; accepted Aug. 26, 2014

Recommended for publication by Associate Editor Jae-Do Park.

${ }^{\dagger}$ Corresponding Author: seuxuyou23@163.com

Tel: +86-13851812862, Nanjing Institute of Technology

*College of Engineering, Nanjing Agriculture University, China

${ }^{* *}$ School of Electrical Engineering, Southeast University, China
PWM rectifier under unbalanced input voltage conditions. Relevant experts and scholars have conducted numerous analyses and research.

Proportional resonant (PR) controller was adopted to realize control of current under two-phase static coordinate that is free from static error [1]-[6]. However, the analysis and design of PR controller are difficult, and the control performance is affected by grid frequency. Furthermore, a DC voltage outer loop PI controller and two PR current inner loop controllers are required. Given that a single-converter structure is adopted, the three-phase grid currents of converter are unsymmetrical, and the workload of the converter switching device is unbalanced. Positive- and negative-sequence dual-current inner loop PI control structures were presented [7]-[11], and static error-free control for the grid positive- and negative-sequence currents could effectively prevent DC voltage fluctuation and grid current distortion. However, the control structure and algorithm are complicated. The structure requires four current inner loop controllers and one voltage outer loop controller, 
namely, five PI controllers. Thus, a large amount of calculation is required. Parameter tuning is also difficult, and the design uses the single-converter structure as well. Therefore, the converter could not achieve symmetrical three-phase currents, thereby leading to unbalanced workload of converter switching devices.

In view of the preceding analysis, the goal of unbalanced control strategy for three-phase PWM rectifier under unbalanced grid voltage conditions lies in the following factors: the stability of DC voltage, the sinusoidal AC current, and the three-phase symmetric currents, which can balance the workload of the switching devices. The traditional control strategy and the topology could not achieve the three control targets simultaneously.

On the basis of the comprehensive analysis of the preceding papers and research achievements, a novel unbalanced one-cycle control (OCC) strategy for dual-converter PWM rectifier under unbalanced voltage conditions is proposed in this paper according to the perspective of the preceding three control targets. The dual-converter PWM rectifier employs two converters, which are called positive-sequence converter and negative-sequence converter. This unbalanced OCC strategy compensates feedback currents of positive-sequence converter via grid negative-sequence voltages and compensates feedback currents of negative-sequence converter via grid positive-sequence voltages. Therefore, the positive-sequence currents flow through the positive-sequence converter, and the negative-sequence currents flow through the negative-sequence converter. The currents of every converter are symmetrical, and the workload of every switching device is balanced. The harmonic of DC voltage and the distortion of line currents of three-phase PWM rectifier have been eliminated effectively under unbalanced voltage conditions. Unlike the above unbalanced control strategies, the proposed unbalanced OCC strategy uses only one conventional outer loop PI controller, which regulates power to be constant and requires no parameter tuning for current inner loops. The calculations for the AC positive- and negative-sequence currents are also not required. Thus, the proposed unbalanced OCC strategy achieves high performance with reduced calculation.

\section{OCC FOR DUAL-CONVERTER THREE-PHASE PWM RECTIFIER}

The topology of OCC for three-phase PWM rectifier is shown in Fig. 1 [12]. In this figure, $e_{\mathrm{a}}, e_{\mathrm{b}}, e_{\mathrm{c}}$ represent the three-phase AC voltages of grid, while $i_{\mathrm{a}}, i_{\mathrm{b}}, i_{\mathrm{c}}$ stand for the grid three-phase AC current; $U_{\mathrm{dc}}$ is the DC voltage, $R$ is the equivalent DC load, and $S_{x y}$ is $(x=\mathrm{a}, \mathrm{b}, \mathrm{c}$ and $y=\mathrm{p}, \mathrm{n})$ the

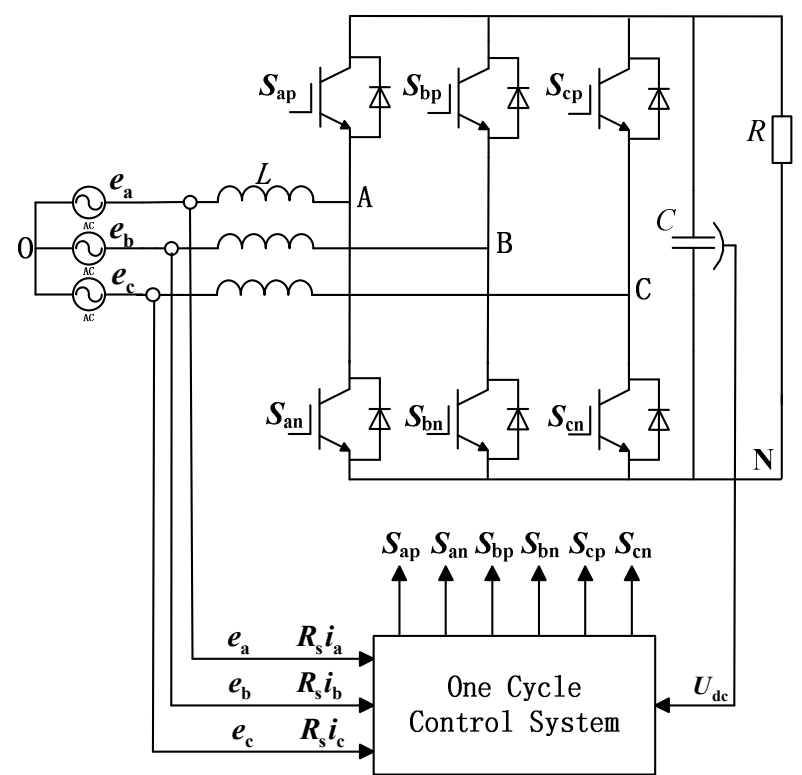

Fig. 1. Topology of OCC for three-phase PWM rectifier.

switching devices of PWM rectifier.

Assuming that grid voltages are under symmetric conditions, the above topology and the traditional OCC system could perform well, and the operating performance would deteriorate sharply under unbalanced grid voltage conditions.

According to the symmetrical component decomposition method, the unbalanced grid voltages could be decomposed into positive- and negative-sequence as well as zero-sequence components. Given that the three-phase PWM rectifier adopts three-wire connection, no zero-sequence components flow through the converter. Therefore, given that the zero-sequence voltages are removed, the unbalanced grid voltages are expressed as follows:

$$
e_{x}^{\prime}=e_{x}-\left(e_{\mathrm{a}}+e_{\mathrm{b}}+e_{\mathrm{c}}\right) / 3=e_{x+}+e_{x-}
$$

where $e_{x^{+}}$and $e_{x^{-}}(x=\mathrm{a}, \mathrm{b}, \mathrm{c})$ denote the positive- and negative-sequence voltage component, respectively.

When the grid voltages are under unbalanced condition, the active power of the rectifier must be a constant value by regulating this active power based on power balance principle to restrain the DC voltage second harmonics of the rectifier and the grid currents distortion. In addition, the relationship between positive- and negative-sequence voltages and currents was derived in [13].

$$
\left\{\begin{array}{l}
{\left[\varphi_{x+}(t)-\theta_{x+}(t)\right]+\left[\varphi_{x-}(t)-\theta_{x-}(t)\right]=180^{\circ}} \\
\frac{\left|e_{x+}\right|}{\left|i_{x+}\right|}=\frac{\left|e_{x-}\right|}{\left|i_{x-}\right|}
\end{array}\right.
$$

where $\varphi_{x+}(t)$ and $\varphi_{x-}(t)$ denote the instantaneous phase of the grid positive- and negative-sequence voltages, respectively, $\theta_{x^{+}}(t)$ and $\theta_{x^{-}}(t)$ represent the instantaneous phase of the grid positive- and negative-sequence current, and $i_{x+}$ and $i_{x^{-}}$are 


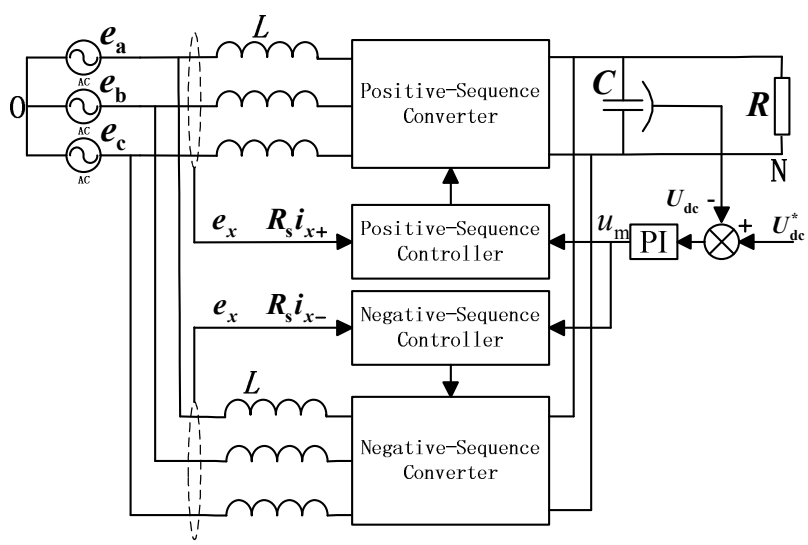

Fig. 2. Topology of unbalanced OCC for dual-converter three-phase PWM rectifier.

the three-phase positive- and negative-sequence currents ( $x=$ $a, b, c)$.

The unbalanced OCC strategy for dual-converter three-phase PWM rectifier, which is shown in Fig. 2, employs the positive-sequence converter to regulate the grid positive-sequence currents, as well as the negative-sequence converter to regulate grid negative-sequence currents.

According to the relationship shown in (3), the unity-power factor of the rectifier can be achieved by controlling the phase of the grid positive-sequence voltages and currents:

$$
\varphi_{+}(t)=\theta_{+}(t)
$$

From the first item of (2) and (3), the phase relationship between the negative-sequence currents and voltages can be derived as follows:

$$
\varphi_{-}(t)-\theta_{-}(t)=180^{\circ}
$$

The grid positive- and negative-sequence voltages and currents are expressed as follows:

$$
\left\{\begin{array}{l}
e_{x+}=R_{\mathrm{e}+} i_{x+} \\
e_{x-}=R_{\mathrm{e}-} i_{x-}
\end{array}\right.
$$

where $R_{\mathrm{e}+}$ and $R_{\mathrm{e}-}$ denote the equivalent positive- and negative-sequence power resistors of three-phase PWM rectifier, respectively. From the second item of (2), (3), and (4), the following can be obtained:

$$
R_{\mathrm{e}-}=-R_{\mathrm{e}+}
$$

If $R_{\mathrm{e}+}=R_{\mathrm{e}}$, then the following can be obtained:

$$
R_{\mathrm{e}-}=-R_{\mathrm{e}}
$$

Three-phase currents of the rectifier can be depicted as follows:

$$
i_{x}=i_{x+}+i_{x-}
$$

Fig. 3 shows the equivalent circuit of the positive or negative converter, which is shown in Fig. $1[12,16]$. In Fig. $3, d_{\mathrm{an}}, d_{\mathrm{bn}}, d_{\mathrm{cn}}$ are the duty ratios for the converter lower bridge arm switches $\mathrm{S}_{\mathrm{an}}, \mathrm{S}_{\mathrm{bn}}, \mathrm{S}_{\mathrm{cn}}$, respectively.

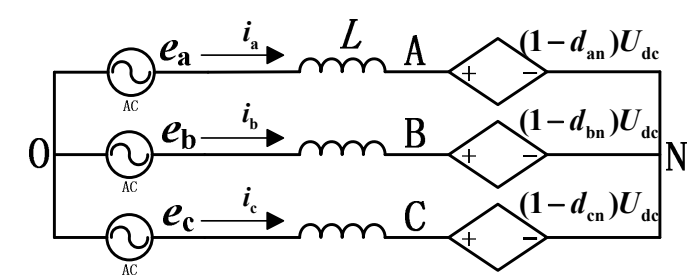

Fig. 3. Equivalent average model of OCC for three-phase PWM rectifier.

The average voltages of rectifier bridge nodes A, B, and C, which are relative to node $\mathrm{N}$, are expressed as follows:

$$
u_{X \mathrm{~N}}=\left(1-d_{x \mathrm{n}}\right) U_{\mathrm{dc}}
$$

where $X=\mathrm{A}, \mathrm{B}, \mathrm{C}, x=\mathrm{a}, \mathrm{b}$, c. Compared with the neutral point $\mathrm{O}$, grid voltage phasor $u_{x O}$ and converter AC side voltage phasor $u_{X O}$ follow that

$$
\dot{u}_{X \mathrm{O}}=\dot{u}_{x \mathrm{O}}-\mathrm{j} \omega L \dot{i}_{x}
$$

According to the analysis in paper [12], the value of the grid side rectifier inductor is actually small (usually approximately $1 \mathrm{mH}$ ), and the fundamental wave voltage drops across the inductance can be ignored. Therefore, the converter $\mathrm{AC}$ side voltages are approximately equal to the grid voltages:

$$
\dot{u}_{X \mathrm{O}} \approx \dot{u}_{x \mathrm{O}} \Rightarrow u_{X \mathrm{O}} \approx u_{x \mathrm{O}}=e_{x}^{\prime}
$$

In Fig. 3, the voltages of nodes A, B, C, node N, and node O must be imposed such that

$$
\left\{\begin{array}{l}
u_{\mathrm{AO}}=u_{\mathrm{AN}}+u_{\mathrm{NO}} \\
u_{\mathrm{BO}}=u_{\mathrm{BN}}+u_{\mathrm{NO}} \\
u_{\mathrm{CO}}=u_{\mathrm{CN}}+u_{\mathrm{NO}}
\end{array}\right.
$$

According to (1), the three-phase converter does not include zero-sequence voltages, and the sum of voltages $u_{\mathrm{AO}}$, $u_{\mathrm{BO}}$, and $u_{\mathrm{CO}}$ is equal to zero, that is, $u_{\mathrm{AO}}+u_{\mathrm{BO}}+u_{\mathrm{CO}}=0$. From (12), we could obtain the voltages between nodes $\mathrm{N}$ and $\mathrm{O}$

$$
u_{\mathrm{NO}}=-\frac{1}{3}\left(u_{\mathrm{AN}}+u_{\mathrm{BN}}+u_{\mathrm{CN}}\right)
$$

When (13), (11), and (9) are substituted into (12), the relationship between the grid voltages and ratios for rectifier lower bridge arm switches can be derived as follows:

$$
\left[\begin{array}{rrr}
-\frac{2}{3} & \frac{1}{3} & \frac{1}{3} \\
\frac{1}{3} & -\frac{2}{3} & \frac{1}{3} \\
\frac{1}{3} & \frac{1}{3} & -\frac{2}{3}
\end{array}\right] \cdot\left[\begin{array}{l}
d_{\mathrm{an}} \\
d_{\mathrm{bn}} \\
d_{\mathrm{cn}}
\end{array}\right]=\frac{1}{U_{\mathrm{dc}}} \cdot\left[\begin{array}{c}
e_{\mathrm{a}}^{\prime} \\
e_{\mathrm{b}}^{\prime} \\
e_{\mathrm{c}}^{\prime}
\end{array}\right]
$$

Given that the matrix of (14) is singular, no unique solution exists. Therefore, the following possible solution exists: 


$$
\left\{\begin{array}{l}
d_{\mathrm{an}}=k_{1}-e_{\mathrm{a}}^{\prime} / U_{\mathrm{dc}} \\
d_{\mathrm{bn}}=k_{1}-e_{\mathrm{b}}^{\prime} / U_{\mathrm{dc}} \\
d_{\mathrm{cn}}=k_{1}-e_{\mathrm{c}}^{\prime} / U_{\mathrm{dc}}
\end{array}\right.
$$

where $k_{1}$ could be a random constant, because the duty ratio $d_{x \mathrm{n}}$ must satisfy the inequality, $0<d_{x \mathrm{n}}<1(x=\mathrm{a}, \mathrm{b}, \mathrm{c})$. When (15) is substituted into this inequality, the following can be obtained:

$$
e_{x}^{\prime} / U_{\mathrm{dc}}<k_{1}<1+e_{x}^{\prime} / U_{\mathrm{dc}}
$$

From (16), to ensure $k_{1}$ is a constant, which would not change along with the grid transient voltages, the following can be acquired:

$$
\max \left(e_{x}^{\prime} / U_{\mathrm{dc}}\right)<\min \left(1+e_{x}^{\prime} / U_{\mathrm{dc}}\right)
$$

From (16) and (17), the range of $k_{1}$ can be derived as follows:

$$
E_{\mathrm{m} x} / U_{\mathrm{dc}}<k_{1}<1-E_{\mathrm{m} x} / U_{\mathrm{dc}}
$$

where $E_{\mathrm{m} x}$ denotes the grid voltage amplitude.

From (18), the relationship between the DC voltage of three-phase rectifier based on OCC and the grid voltage amplitude can be expressed as follows:

$$
E_{\mathrm{m} x} / U_{\mathrm{dc}}<1-E_{\mathrm{m} x} / U_{\mathrm{dc}} \Rightarrow U_{\mathrm{dc}}>2 E_{\mathrm{m} x}
$$

From (18), the parameter $k_{1}$ could be any numerical value in the range of $\left[E_{\mathrm{m} x} / U_{\mathrm{dc}}, 1-E_{\mathrm{m} x} / U_{\mathrm{dc}}\right]$, but generally for the sake of increasing application, scope for $k_{1}, k_{1}$ should be assigned to the middle point value of the range in (18):

$$
k_{1}=0.5
$$

Equations (18) and (19) indicate that the essential condition for the OCC system for three-phase PWM rectifier to operate correctly is that the DC voltage is more than double the grid phase voltage amplitude, and then the parameter $k_{1}$ could have a solution.

\section{OCC STRATEGY FOR Positive-SEQUENCE CONVERTER OF PWM RECTIFIER}

Only positive-sequence current $i_{x+}$ should flow through the positive-sequence converter to keep three-phase currents of the positive-sequence converter symmetrical and sinusoidal. Thus, the relationship between voltages and currents of the positive-sequence converter is expressed as follows:

$$
R_{\mathrm{e}} i_{x+}=e_{x+}
$$

According to (21), the control system compensates feedback currents of the positive-sequence converter by grid negative-sequence voltages under unbalanced grid voltage conditions

$$
e_{x-}+R_{\mathrm{e}} i_{x^{+}}=e_{x-}+e_{x^{+}}=e_{x}^{\prime}
$$

According to (22), the currents of the positive-sequence converter are straightforwardly derived as follows:

$$
i_{x+}=\frac{e_{x}^{\prime}}{R_{\mathrm{e}}}-\frac{e_{x-}}{R_{\mathrm{e}}}
$$

If $R_{\mathrm{s}}$ is the equivalent current sampling resistance [14], [15], then Equation (23) could be expressed as follows:

$$
R_{\mathrm{s}} i_{x+}=\frac{R_{\mathrm{s}} e_{x}^{\prime}}{R_{\mathrm{e}}}-\frac{R_{\mathrm{s}} e_{x-}}{R_{\mathrm{e}}}
$$

For the positive-sequence converter, Equation (15) could be rewritten as follows:

$$
\left\{\begin{array}{l}
d_{\mathrm{an}+}=k_{1}-e_{\mathrm{a}}^{\prime} / U_{\mathrm{dc}} \\
d_{\mathrm{bn}+}=k_{1}-e_{\mathrm{b}}^{\prime} / U_{\mathrm{dc}} \\
d_{\mathrm{cn}+}=k_{1}-e_{\mathrm{c}}^{\prime} / U_{\mathrm{dc}}
\end{array}\right.
$$

where $d_{x n+}$ is the duty ratio for the positive-sequence converter lower bridge arm switches. When (25) is substituted into (24), the following is produced:

$$
R_{\mathrm{s}} i_{x+}=\frac{k_{1} R_{\mathrm{s}}}{R_{\mathrm{e}}} U_{\mathrm{dc}}\left(1-\frac{d_{x \mathrm{n}+}}{k_{1}}\right)-\frac{R_{\mathrm{s}}}{R_{\mathrm{e}}} e_{x-}
$$

When $d_{x n^{+}}=1-t_{x p+} / T_{\mathrm{s}}$ is substituted into (26), the following can be obtained:

$$
R_{\mathrm{s}} i_{x+}=\frac{k_{1} R_{\mathrm{s}}}{R_{\mathrm{e}}} U_{\mathrm{dc}}\left(\frac{t_{x \mathrm{p}+}}{k_{1} T_{\mathrm{s}}}-1-\frac{e_{x-}}{k_{1} U_{\mathrm{dc}}}\right)
$$

where $T_{\mathrm{s}}$ denotes the switching period of switches, and $t_{x \mathrm{p}+}$ is the turn-on time of each positive-sequence converter upper bridge arm switch in one switching period. From (27), OCC key equations for the positive-sequence converter can be obtained:

$$
\left\{\begin{array}{l}
R_{\mathrm{s}} i_{\mathrm{a}}=u_{\mathrm{m}+}\left(\frac{t_{\mathrm{ap}^{+}}}{\tau_{+}}-1-\frac{e_{\mathrm{a}-}}{U_{\mathrm{dc}} \cdot k_{1}}\right) \\
R_{\mathrm{s}} i_{\mathrm{b}}=u_{\mathrm{m}+}\left(\frac{t_{\mathrm{bp}+}}{\tau_{+}}-1-\frac{e_{\mathrm{b}-}}{U_{\mathrm{dc}} \cdot k_{1}}\right) \\
R_{\mathrm{s}} i_{\mathrm{c}}=u_{\mathrm{m}+}\left(\frac{t_{\mathrm{cp}+}}{\tau_{+}}-1-\frac{e_{\mathrm{c}-}}{U_{\mathrm{dc}} \cdot k_{1}}\right)
\end{array}\right.
$$

where

$$
\left\{\begin{array}{l}
u_{\mathrm{m}+}=\frac{R_{\mathrm{s}} k_{1}}{R_{\mathrm{e}}} U_{\mathrm{dc}} \\
\tau_{+}=k_{1} T_{\mathrm{s}}
\end{array}\right.
$$

where $u_{\mathrm{m}+}$ denotes the positive-sequence DC error control voltage, which is used to control output power for rectifier; $\tau_{+}$stands for the integral time constant of integrator in OCC system, and its value is half the switching period; and $-u_{\mathrm{m}+} e_{x-} /\left(U_{\mathrm{dc}} k_{1}\right)$ is the grid negative-sequence voltage compensation item. According to (28), the OCC system structure for the positive-sequence converter can be shown as in Fig. 4.

\section{OCC STRATEGY FOR NEGATIVE-SEQUENCE CONVERTER OF PWM RECTIFIER}

Only negative-sequence current $i_{x-}$ should flow through the negative-sequence converter to keep three-phase currents of 


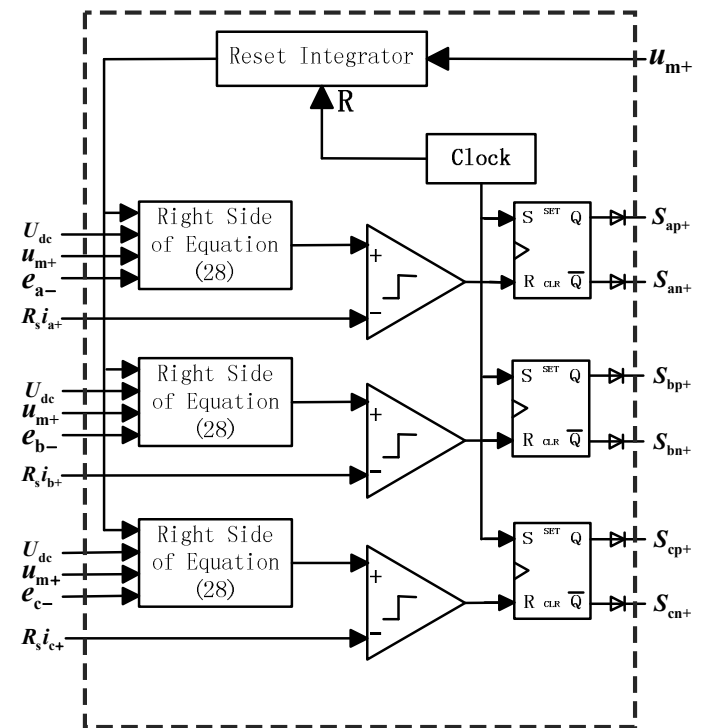

Fig. 4. Unbalanced $\mathrm{OC} \bar{C}$ system for positive-sequence converter.

the positive-sequence converter symmetrical and similar. Thus, the relationship between voltages and currents for the negative-sequence converter is expressed as follows:

$$
R_{\mathrm{e}-} i_{x-}=-R_{\mathrm{e}} i_{x-}=e_{x-}
$$

According to (30), the control system compensates feedback currents of negative-sequence converter via grid positive-sequence voltages under unbalanced grid voltage condition:

$$
e_{x+}-R_{\mathrm{e}} i_{x-}=e_{x+}+e_{x-}=e_{x}^{\prime}
$$

According to (31), the currents of the negative-sequence converter are straightforwardly derived as follows:

$$
i_{x-}-\frac{e_{x+}}{R_{\mathrm{e}}}=-\frac{e_{x}^{\prime}}{R_{\mathrm{e}}}
$$

If $R_{\mathrm{S}}$ is the equivalent current sampling resistance, then Equation (32) could be expressed as follows:

$$
\left\{\begin{array}{l}
R_{\mathrm{s}} i_{x-}-\frac{R_{\mathrm{s}}}{R_{\mathrm{e}}} e_{x+}=-\left(k_{3} R_{\mathrm{s}} e_{x}^{\prime}-k_{2} R_{\mathrm{s}} U_{\mathrm{dc}} \frac{e_{x}^{\prime}}{U_{\mathrm{dc}}}\right) \\
k_{3}-k_{2}=1 / R_{\mathrm{e}}
\end{array}\right.
$$

where $k_{2}$ and $k_{3}$ are positive constants that are used to control power for negative-sequence converter and maximum grid currents, respectively. For the negative-sequence converter, Equation (15) could be rewritten as follows:

$$
\left\{\begin{array}{l}
d_{\mathrm{ap}-}=1-k_{1}+e_{\mathrm{a}}^{\prime} / U_{\mathrm{dc}} \\
d_{\mathrm{bp}-}=1-k_{1}+e_{\mathrm{b}}^{\prime} / U_{\mathrm{dc}} \\
d_{\mathrm{cp}-}=1-k_{1}+e_{\mathrm{c}}^{\prime} / U_{\mathrm{dc}}
\end{array}\right.
$$

where $d_{x p-}$ is the duty ratio for the negative-sequence converter upper bridge arm switches. When (34) is substituted into (33), the following can be obtained:

$$
R_{\mathrm{s}} i_{x-}-\frac{R_{\mathrm{s}}}{R_{\mathrm{e}}} e_{x+}=-k e_{x}^{\prime}+\left(k-\frac{R_{\mathrm{s}}}{R_{\mathrm{e}}}\right) U_{\mathrm{dc}}\left[d_{x \mathrm{p}-}-\left(1-k_{1}\right)\right]
$$

where $k$ is similar to $k_{3}$ and used to restrain maximum grid currents. Then, substituting the duty ratio $d_{x \mathrm{p}^{-}}=t_{x^{-}} / T_{\mathrm{s}}$ into (35) can obtain the following control key equation:

$$
k e_{x-}+R_{\mathrm{s}} i_{x-}=\left(k-\frac{R_{\mathrm{s}}}{R_{\mathrm{e}}}\right)\left(1-k_{1}\right) U_{\mathrm{dc}}\left[\frac{t_{x p-}}{\left(1-k_{1}\right) T_{\mathrm{s}}}-1-\frac{e_{x+}}{U_{\mathrm{dc}}\left(1-k_{1}\right)}\right]
$$

where $T_{\mathrm{s}}$ denotes the switching period of switches, and $t_{x \mathrm{p}-}$ is the turn-on time of each negative-sequence converter upper bridge arm switch in one switching period. From (36), the OCC key equation for the negative-sequence converter can be obtained:

$$
\left\{\begin{array}{l}
k e_{\mathrm{a}-}+R_{\mathrm{s}} i_{\mathrm{a}-}=u_{\mathrm{m}-}\left[\frac{t_{\mathrm{ap}-}}{\tau_{-}}-1-\frac{e_{\mathrm{a}+}}{U_{\mathrm{dc}}\left(1-k_{1}\right)}\right] \\
k e_{\mathrm{b}-}+R_{\mathrm{s}} i_{\mathrm{b}-}=u_{\mathrm{m}-}\left[\frac{t_{\mathrm{bp}-}}{\tau_{-}}-1-\frac{e_{\mathrm{b}+}}{U_{\mathrm{dc}}\left(1-k_{1}\right)}\right] \\
k e_{\mathrm{c}-}+R_{\mathrm{s}} i_{\mathrm{c}-}=u_{\mathrm{m}-}\left[\frac{t_{\mathrm{cp}-}}{\tau_{-}}-1-\frac{e_{\mathrm{c}+}}{U_{\mathrm{dc}}\left(1-k_{1}\right)}\right]
\end{array}\right.
$$

where

$$
\left\{\begin{array}{l}
u_{\mathrm{m}-}=\left(k-\frac{R_{\mathrm{s}}}{R_{\mathrm{e}}}\right)\left(1-k_{1}\right) U_{\mathrm{dc}} \\
\tau_{-}=\left(1-k_{1}\right) T_{\mathrm{s}}
\end{array}\right.
$$

where $u_{\mathrm{m}-}$ denotes the negative-sequence DC error control voltage, which is used to control output power for rectifier. $\tau_{-}$ is the integral time constant of integrator of OCC system, and its value is half of switching period, and $-u_{\mathrm{m}-} e_{x+} /\left(U_{\mathrm{dc}}\left(1-k_{1}\right)\right)$ is the grid positive-sequence voltage compensation item. According to (37), the OCC system structure for the negative-sequence converter can be shown in Fig. 5.

According to (28) and (37), the positive and negative-sequence converters adopt the same bipolar OCC strategy, and the switching operations of positive- and negative-sequence converter switches are nearly at the same time based on Figs. 4 and 5. Therefore, the high-frequency circulation currents between the positive-sequence converter and the negative-sequence converter can be eliminated [16].

\section{UNBALANCED OCC SYSTEM FOR DUAL-CONVERTER PWM RECTIFIER}

Grid positive- and negative-sequence voltages could be decomposed under two-phase stationary coordinate system from the retained voltage, which is described by (39).

$$
\left[\begin{array}{c}
e_{\alpha+} \\
e_{\beta+} \\
e_{\alpha-} \\
e_{\beta-}
\end{array}\right]=\frac{1}{2}\left[\begin{array}{rrrr}
1 & 0 & 0 & -1 \\
0 & 1 & 1 & 0 \\
1 & 0 & 0 & 1 \\
0 & 1 & -1 & 0
\end{array}\right]\left[\begin{array}{llll}
e_{\alpha} & e_{\beta} & e_{\alpha}^{\perp} & e_{\beta}^{\perp}
\end{array}\right]^{\mathrm{T}}
$$

where $e_{\alpha}$ and $e_{\beta}$ are $\alpha$ and $\beta$ axis coordinate components of the 


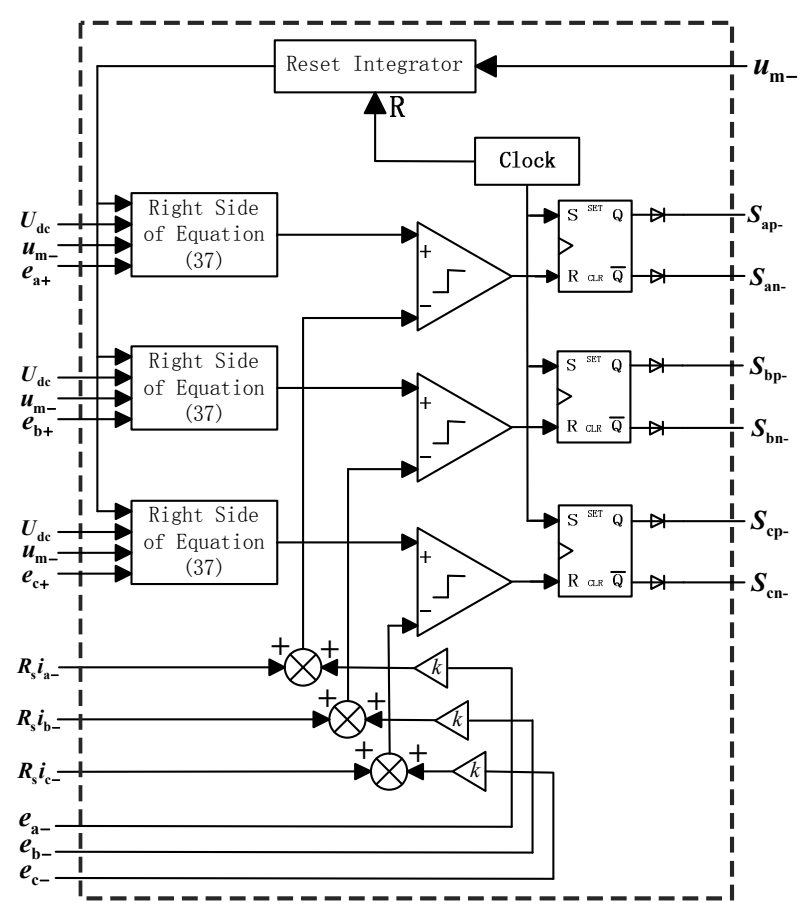

Fig. 5. Unbalanced OCC system for negative-sequence converter.

grid voltages, respectively, and $e_{\alpha}{ }^{\perp}$ and $e_{\beta}{ }^{\perp}$ denote the $\alpha$ and $\beta$ axis coordinate components, which delay one-fourth power period. The transient values of the grid positive- and negative-sequence voltages under three-phase stationary coordinate are derived as follows:

$$
\begin{aligned}
& {\left[\begin{array}{l}
e_{\mathrm{a}^{+}} \\
e_{\mathrm{b}+} \\
e_{\mathrm{c}+}
\end{array}\right]=\left[\begin{array}{rc}
1 & 0 \\
-\frac{1}{2} & \frac{\sqrt{3}}{2} \\
-\frac{1}{2} & -\frac{\sqrt{3}}{2}
\end{array}\right]\left[\begin{array}{l}
e_{\alpha+} \\
e_{\beta+}
\end{array}\right]} \\
& {\left[\begin{array}{l}
e_{\mathrm{a}-} \\
e_{\mathrm{b}-} \\
e_{\mathrm{c}-}
\end{array}\right]=\left[\begin{array}{rr}
1 & 0 \\
-\frac{1}{2} & \frac{\sqrt{3}}{2} \\
-\frac{1}{2} & -\frac{\sqrt{3}}{2}
\end{array}\right]\left[\begin{array}{l}
e_{\alpha-} \\
e_{\beta-}
\end{array}\right]}
\end{aligned}
$$

According to the characteristics of the dual-converter PWM rectifier, the positive- and negative-sequence converters must share the same PI outer loop voltage controller. Therefore, the error control voltage of the positive sequence $u_{\mathrm{m}+}$ is equal to the error control voltage of the negative sequence $u_{\mathrm{m}-}$. According to (29) and (38), $u_{\mathrm{m}+}$ can be made equal to $u_{\mathrm{m}-}$ by adjusting the parameters $k, R_{\mathrm{s}}$, and $R_{\mathrm{e}}$. If $u_{\mathrm{m}+}=u_{\mathrm{m}-}=u_{\mathrm{m}}$ and given that $k_{1}=0.5$, namely, $k_{1}=1-k_{1}$, then parameter $k$ is expressed as follows:

$$
k=\frac{2 R_{\mathrm{s}}}{R_{\mathrm{e}}}
$$

The mathematical relationship between error control voltage $u_{\mathrm{m}}$ and DC voltage $U_{\mathrm{dc}}$ can be derived as follows:

$$
u_{\mathrm{m}}=\frac{k}{4} U_{\mathrm{dc}}
$$

From (43), PI controller can be employed to control the DC voltage for the unbalanced OCC system, and the control equation for DC voltage is expressed as follows:

$$
u_{\mathrm{m}}=\left(K_{\mathrm{P}}+K_{\mathrm{I}} \int \mathrm{d} t\right)\left(U_{\mathrm{dc}}^{*}-U_{\mathrm{dc}}\right)
$$

where $U_{\text {dc }}^{*}$ denotes the set value of DC voltage for rectifier, $U_{\mathrm{dc}}$ is the rectifier feedback DC voltage, and $K_{\mathrm{P}}$ and $K_{\mathrm{I}}$ are the proportionality and integral coefficient for PI controller, respectively. From Fig. 2 and (43), the following can be obtained:

$$
k=\frac{4 u_{\mathrm{m}}}{U_{\mathrm{dc}}^{*}}
$$

According to (28) and Fig. 4, the feedback currents of the positive-sequence converter, which are the positive-sequence currents, must be transmitted to the negative inputs of the comparator of the positive-sequence controller to ensure that the three-phase currents, which flow through the positive-sequence converter, are symmetric. According to OCC theory, the positive-sequence currents of the positive-sequence converter could be used to cancel out the grid positive-sequence voltages. The positive- and negative-sequence voltages exist under unbalanced grid voltage conditions simultaneously, and the grid negative-sequence voltage compensation component is transmitted to the positive inputs of comparator of positive-sequence controller to cancel out the negative-sequence voltages. Therefore, the feedback currents of the positive-sequence converter should be compensated by negative-sequence components of grid voltages. Similarly, from (37) and Fig. 5, the feedback currents of the negative-sequence converter, which are the negative-sequence currents, must be transmitted to the positive inputs of comparator of positive-sequence controller to ensure that the three-phase currents, which flow through the negative-sequence converter, are symmetric. According to (6), the positive-sequence converter serves as a rectifier, whereas the negative-sequence converter serves as a grid-connected inverter that inverts the second harmonic power of the DC side back to grid in form of sinusoidal currents. Therefore, the second harmonic waves of DC voltage and distortion of line currents can be eliminated.

According to (28) and (37), the positive- and negative-sequence currents flow through the positive- and negative-sequence converters, respectively, and the unbalanced factor for the grid voltages is defined by [17]:

$$
V U F=\frac{\left|U_{-}\right|}{\left|U_{+}\right|}=\sqrt{\frac{6\left(U_{\mathrm{ab}}^{2}+U_{\mathrm{bc}}^{2}+U_{\mathrm{ca}}^{2}\right)}{\left(U_{\mathrm{ab}}+U_{\mathrm{bc}}+U_{\mathrm{ca}}\right)^{2}}-2}
$$




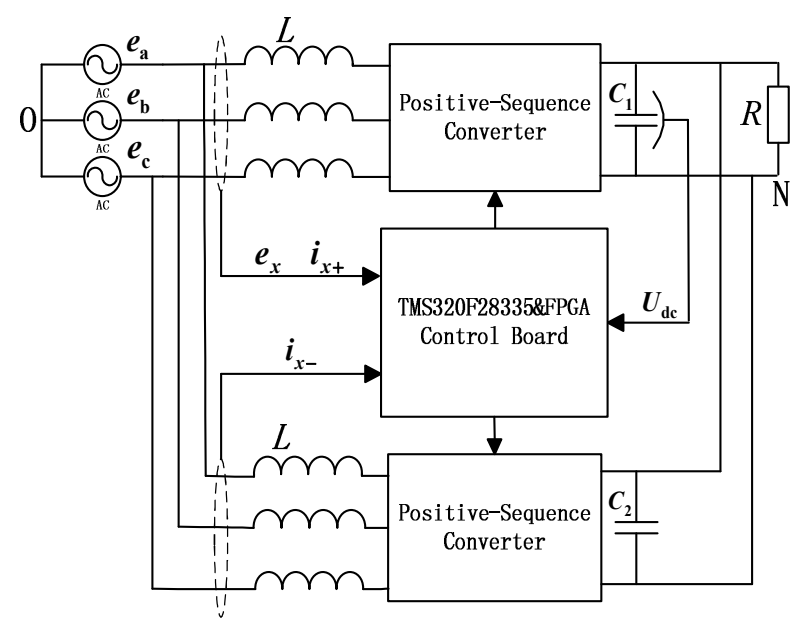

Fig. 6. Experiment system of unbalanced OCC for dual-converter three-phase PWM rectifier.

where $U_{+}$and $U_{-}$denote the grid positive- and negative-sequence RMS voltages, respectively, whereas $U_{\mathrm{ab}}$, $U_{\mathrm{bc}}$, and $U_{\mathrm{ca}}$ are the grid RMS line voltages. The relationship between positive- and negative-sequence currents can be derived as follows:

$$
\left|i_{x-}\right|=V U F\left|i_{x+}\right|
$$

According to (47), the negative-sequence converter is $V U F$ times the capacity of design and the rated currents of switching devices and inductance of positive-sequence converter in engineering applications. To achieve consistency, the inductance of negative-sequence converter should be designed to be $1 / V U F$ times the value of the inductance of the positive-sequence converter. The reduction in the rated currents of inductance of negative-sequence converter could reduce the size of inductor magnetic core and then greatly reduce the cost. For example, if the unbalanced factor $V U F$ is $10 \%$ for the design, then the negative-sequence converter is $10 \%$ the capacity of the positive-sequence converter. In addition, the cost of negative-sequence converter is greatly reduced. According to (47), because $i_{x-}$ is less than $i_{x+}$, the loss of negative-sequence converter is also less than positive-sequence converter. Therefore, the extra loss caused by the negative-sequence converter increases slightly. In particular, when the grid voltage is under slightly unbalanced condition and $V U F$ is nearly zero, the current of the negative-sequence converter remains nearly zero, and the loss increases slightly.

\section{EXPERIMENTAL RESULTS}

A three-phase dual-converter PWM rectifier based on unbalanced OCC strategy is designed in this study to verify the validity of the theoretical analysis and research. The control system for the rectifier adopts the digital signal processor (DSP) TMS320F28335, and the capacity of the

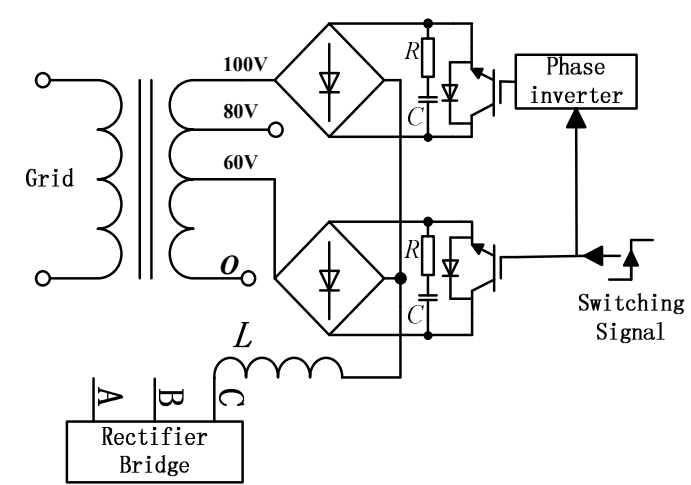

Fig. 7. Voltage sag generator of Phase C.

TABLE I

EXPERIMENTAL PARAMETERS FOR THREE-PHASE PWM RECTIFIER

\begin{tabular}{|c|c|c|}
\hline Given DC voltage & $U_{\mathrm{dc}}^{*}$ & $250 \mathrm{~V}$ \\
\hline Load resistor & $R$ & $20 \Omega$ \\
\hline \multirow{2}{*}{$\begin{array}{l}\text { Filter inductor of positive- and } \\
\text { negative-sequence converter }\end{array}$} & $L_{+}$ & $1.2 \mathrm{mH}$ \\
\hline & $L_{-}$ & $5 \mathrm{mH}$ \\
\hline DC side filter capacitor & $C$ & $1360 \mathrm{uF}$ \\
\hline \multirow{2}{*}{ PI controller parameters } & $K_{\mathrm{P}}$ & 1.6 \\
\hline & $K_{\mathrm{I}}$ & 100 \\
\hline \multirow[t]{2}{*}{ OCC system parameters } & $R_{\mathrm{S}}$ & $0.5 \Omega$ \\
\hline & $k$ & 0.2 \\
\hline RS flip-flop clock frequency & $f_{\mathrm{s}}$ & $12.8 \mathrm{kHz}$ \\
\hline \multirow{2}{*}{$\begin{array}{l}\text { Integral time constant of the } \\
\text { integrator }\left(\tau_{+}=\tau_{-}=\tau=k_{1} T_{\mathrm{s}}\right)\end{array}$} & $\tau_{+}$ & $0.0390625 \mathrm{~ms}$ \\
\hline & $\bar{\tau} \tau_{-}$ & $0.0390625 \mathrm{~ms}$ \\
\hline
\end{tabular}

rectifier is designed as $5 \mathrm{kVA}$. The experimental system structure is shown in Fig. 6.

The control software was completed by TMS320F28335 DSP. Moreover, the digital integrator, data comparator, RS flip-flop, and clock generator were completed by using a field programmable gate array. On the basis of the laboratory prototype, we conducted research on the conventional rectifier based on balanced OCC strategy and dual-converter rectifier based on unbalanced OCC strategy.

The grid unbalanced fault was simulated by voltage sag generator, which is shown in Fig. 7. The voltage sag generator employed a three-phase transformer with $9000 \mathrm{VA}$ capacity. Each phase of the transformer output windings had three taps, and the output voltage amplitudes of every tap were 100,80 , and $60 \mathrm{~V}$.

The grid was initially under balanced voltage conditions, and three-phase voltage amplitudes were all $100 \mathrm{~V}$. After the rectifier was running stably, we simulated an unbalanced fault by using voltage sag generator. Moreover, the voltage amplitude of Phase A remained $100 \mathrm{~V}$, the voltage amplitude of Phase B dropped to $80 \mathrm{~V}$, and the voltage amplitude of Phase C dropped to $60 \mathrm{~V}$. Table I lists the experimental parameters for the three-phase PWM rectifier, and the experimental results are shown in Fig. 8. 


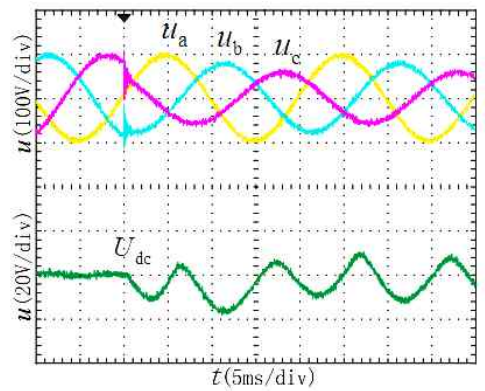

(a) DC voltage waveforms of PWM rectifier based on conventional balanced OCC strategy under Phase B and Phase C voltage sag.

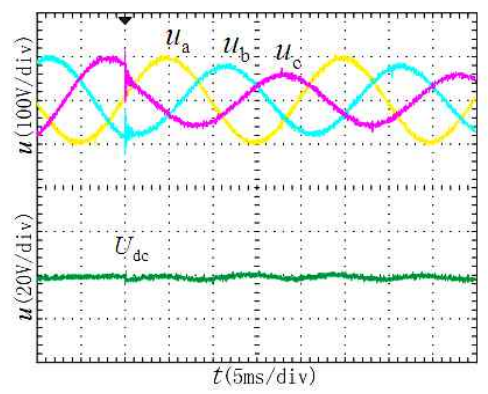

(c) DC voltage waveforms of single-converter PWM rectifier based on PR controller under Phase B and Phase C voltage sag.

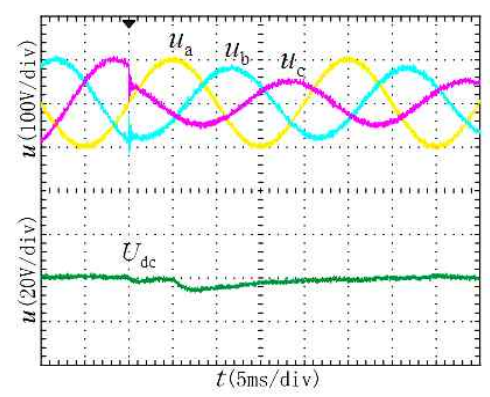

(e) DC voltage waveforms of dual-converter PWM rectifier based on unbalanced OCC strategy under Phase B and Phase C voltage sag.

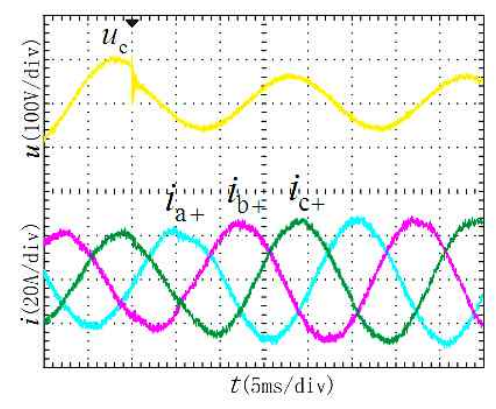

(g) Three-phase current waveforms of positive-sequence converter based on unbalanced OCC strategy under Phase B and Phase $\mathrm{C}$ voltage sag.

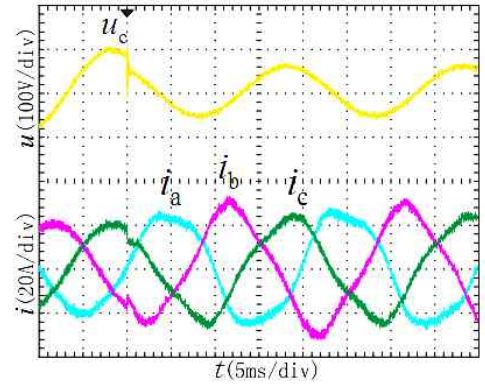

(b) Three-phase current waveforms of PWM rectifier based on conventional balanced OCC strategy under Phase B and Phase C voltage sag.

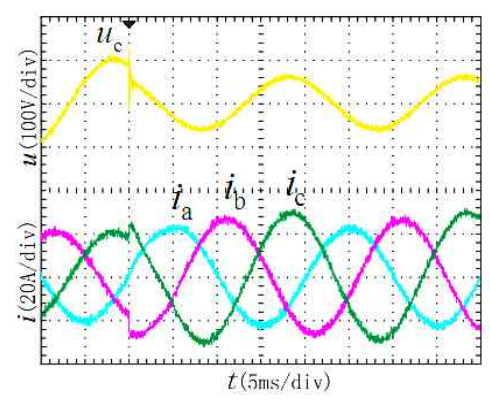

(d) Three-phase current waveforms of single-converter PWM rectifier based on PR controller under Phase B and Phase C voltage sag.

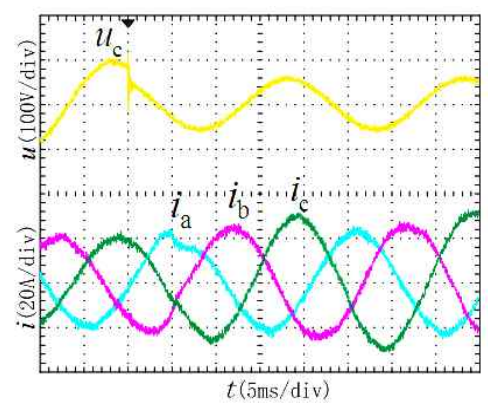

(f) Three-phase current waveforms of dual-converter PWM rectifier based on unbalanced OCC strategy under Phase B and Phase $\mathrm{C}$ voltage sag.

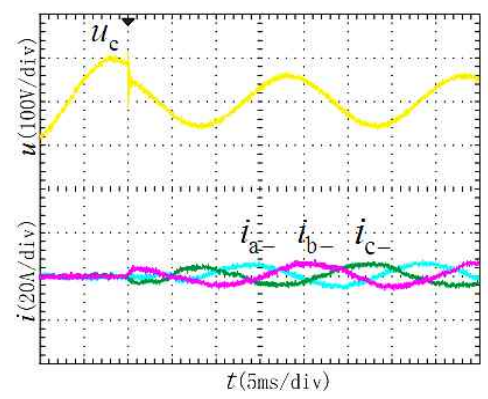

(h) Three-phase current waveforms of negative-sequence converter based on unbalanced OCC strategy under Phase B and Phase $\mathrm{C}$ voltage sag.

Fig. 8. Experimental waveforms of three-phase PWM rectifier under unbalanced grid voltage conditions. 
The experimental waveforms of DC voltage and AC currents of the conventional rectifier based on balanced OCC strategy under unbalanced voltage conditions are shown in Figs. 8(a) and 8(b), respectively, where the second harmonic waves of DC voltage and distortion of line currents appeared. The total harmonic distortion (THD) of the AC currents is nearly $13 \%$, and the oscillation of DC voltage is approximately $19 \mathrm{Vpp}$. The experimental waveforms of DC voltage and $\mathrm{AC}$ currents of the single-converter rectifier based on PR controller under unbalanced voltage conditions are shown in Figs. 8(c) and 8(d), respectively, where the DC voltage oscillation is approximately $4 \mathrm{Vpp}$ in steady state, which is less than that of the conventional rectifier. The line current is nearly sinusoidal, and the distortion is restrained. However, the line current is unsymmetrical. Thus, the workload of every switching device is unbalanced, and the power rating of every switching device cannot be used to the fullest. The experimental waveform of the DC voltage of the dual-converter rectifier based on unbalanced OCC strategy under unbalanced voltage conditions is shown in Fig. 8(e), where the DC voltage enters the steady state rapidly with a slight oscillation, which is only approximately 2 Vpp. Fig. 8(f) shows the AC line currents of the dual-converter rectifier, which are the sum of the positive- and negative-sequence currents under unbalanced voltage conditions. The AC line currents of the dual-converter rectifier are not symmetric but sinusoidal. The three-phase AC current waves of the positiveand negative-sequence converter are shown in Figs. 8(g) and 8(h), where the three-phase AC currents are all symmetric sinusoidal waveforms. The distortion of line currents is eliminated, and the THD is only approximately $2.5 \%$. The power factor is approximately 0.97 , and the dual-converter PWM rectifier runs nearly in unity-power factor state.

\section{CONCLUSIONS}

An unbalanced OCC strategy for dual-converter three-phase PWM rectifier based on grid positive- and negative-sequence voltage feedforward compensation was proposed in this paper to eliminate the second harmonic waves of DC voltage and distortion of line currents for a three-phase PWM rectifier under unbalanced input grid voltage conditions. The unbalanced OCC mathematical model for the positive-sequence converter based on the grid negative-sequence voltage feedforward compensation and the model for the negative-sequence converter based on the grid positive-sequence voltage feedforward compensation were presented. Moreover, the current control and parameter tuning method was investigated. The experimental results indicate that unlike other unbalanced control strategies, the proposed unbalanced OCC strategy for dual-converter three-phase PWM rectifier needs no calculation for the positive- and negative-sequence currents and no designation for current inner loop controller. Therefore, the control system for the dual-converter three-phase rectifier only has one PI controller, and the control structure is simplified. The second harmonic waves of DC voltage and distortion of AC line currents of rectifier are eliminated, and the harmonic pollution to grid is reduced greatly under unbalanced grid voltage conditions. Moreover, the positive- and negative-sequence converters both achieved symmetrical three-phase sinusoidal currents, which balance the workload of all the switching devices of the converters, and high-power factor can be achieved. With the proposed dual-converter topology and the corresponding unbalanced control strategy, the performance of the three-phase PWM rectifier is ultimately optimized under unbalanced grid voltage conditions.

\section{REFERENCES}

[1] L. Moran, P. D. Ziogas, and G. Joos, "Design aspects of synchronous PWM rectifier-inverter systems under unbalanced input voltage conditions," IEEE Trans. Ind. Appl., Vol. 28, No. 6, pp. 1286-1293, Nov./Dec. 1992.

[2] D. Roiu, R. I. Bojoi, R. I. Limongi, and A. Tenconi, "New stationary frame control scheme for three-Phase PWM rectifiers under unbalanced voltage dips conditions," IEEE Trans. Ind. Appl., Vol. 46 No. 1, pp. 268-277, Jan./Feb. 2010.

[3] Z. Li, P. Wang, Y. H. Li, H. Zhu, and F. Gao, "Control of three-phase PWM rectifier under unbalanced input voltage conditions without sequential component extraction," in Proc., PEMC, pp. T2-179-T2184, 2010.

[4] J. B. Hu, Y. K. He, L. Xu, and B. W. Williams, "Improved control of DFIG systems during grid unbalance using PI-R current regulators," IEEE Trans. Ind. Electron., Vol. 56, No. 2, pp. 439-451, Feb. 2009.

[5] J. G. Hwang, P. W. Lehn, and M. Winkelnkemper, "Control of grid connected AC-DC converters with minimized DC link capacitance under unbalanced grid voltage condition," in Proc., IEEE EPE, pp. 1-10, 2007.

[6] I. Etxeberria-Otadui, U. Viscarret, M. Caballero, A. Rufer, and S. Bacha, "New optimized PWM VSC control structures and strategies under unbalanced voltage transients," IEEE Trans. Ind. Electron., Vol. 54, No. 5, pp. 2902-2914, Oct. 2007.

[7] X. H. Wu, K. P. Sahib, and J. X. Xu, "Supply-side current harmonics control of three phase PWM boost rectifiers under distorted and unbalanced supply voltage conditions," in Proc. IEEE PEDS, pp. 647-652, 2007.

[8] Y. Suh and T. A. Lipo, "Control scheme in hybrid synchronous stationary frame for PWM AC/DC converter under generalized unbalanced operating conditions," IEEE Trans. Ind. Appl., Vol. 42, No. 3, pp. 825-835, May/Jun. 2006.

[9] Y. Suh and T. A. Lipo, "Modeling and analysis of instantaneous active and reactive power for PWM AC/DC converter under generalized unbalanced grid," IEEE Trans. Power Del., Vol. 21, No. 3, pp. 1530-1540, Jul. 2006.

[10] H.-S. Song, I.-W. Joo, and K. Nam, "Source voltage sensorless estimation scheme for PWM rectifiers under unbalanced conditions," IEEE Trans. Ind. Electron., Vol. 50, No. 6, pp. 1238-1245, Dec. 2003. 
[11] H.-S. Song and K. Nam, "Dual current control scheme for PWM converter under unbalanced input voltage conditions," IEEE Trans. Ind. Electron., Vol. 46, No. 5, pp. 953-959, Oct. 1999.

[12] C. Qiao and K. M. Smedley, "Unified constant-frequency integration control of three-phase standard bridge boost rectifiers with power-factor correction," IEEE Trans. Ind. Electron., Vol. 50, No. 1, pp. 100-107, Feb. 2003.

[13] A. V. Stankovic and T. A.Lipo, "A novel control method for input output harmonic elimination of the PWM Boost type rectifier under unbalanced operating conditions," IEEE Trans. Power Electron., Vol. 16, No. 5, pp. 603-611, Sep. 2001.

[14] C. Qiao and K. M. Smedley, "A general three-phase PFC controller for rectifiers with a parallel-connected dual Boost topology," IEEE Trans. Power Electron., Vol. 17, No. 6, pp. 925-934, Nov. 2002.

[15] C. Qiao and K. M. Smedley, "Three-phase grid-connected inverters interface for alternative energy sources with unified constant-frequency integration control," in Proc. IEEE IAS, pp. 2675-2682, Sep./Oct. 2001.

[16] Y. Chen and K. M. Smedley, "Parallel operation of one-cycle controlled three-phase PFC rectifiers," IEEE Tans. Ind. Electron., Vol. 54, No. 6, pp. 3217-3224, Dec. 2007.

[17] H. Markiewicz and A. Klajn, "European Standard-Voltage characteristics in Public Distribution System, EN50160," in Proc. European Committee for standardization (EN), pp. 1-12, Apr. 2008.

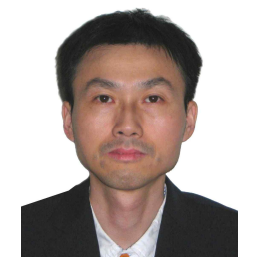

You Xu was born in Jiangsu, China. He received his B.S. degree in Electrical Engineering from China University of Mining and Technology, Xuzhou, China, in 2002, and his M.S. and Ph.D. degrees in Electrical Engineering from Southeast University, Nanjing, China, in 2007 and 2013, respectively. $\mathrm{He}$ is now a lecturer in the School of Automation, Nanjing Institute of Technology. His current research interests include electric power converters for distributed generation, fault ride through for grid-connected inverters, and the application of embedded system for power electronics in power systems.

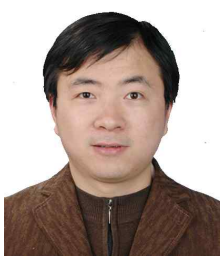

Qingjie Zhang was born in Jiangsu, China. He received his B.S. in Electrical Engineering from Yancheng Institute of Technology, China, in 2004, and his M.S. in Electrical engineering from Southeast University, China, in 2007. He is now pursuing his Ph.D. in Electrical Engineering at Southeast University, Nanjing, China. He is a lecturer in the College of Engineering, Nanjing Agricultural University. His current interests include power electronics for renewable energy generation, power system stability control, and microgrid technology.

Kai Deng was born in Jiangsu, China. He received his B.S. from Nanjing Agricultural University, Nanjing, China, in 2009, and his M.S. from Beijing Institute of Technology, Beijing, China, in 2012, both in Electrical Engineering. He is now pursuing his Ph.D. in Electrical Engineering in Southeast University, Nanjing, China. His current research interests include Z-source inverters, digital control of power converters, and system integration of modular power converters. 